Rev Inv Vet Perú 2004; 15 (2): 170-173

\title{
Comunicación
}

\section{TOXOPLASMOSIS EN ALPACAS DE LA SIERRA ALTOANDINA}

\author{
Francisco Suárez A. ${ }^{1}$, Wally Flores G. ${ }^{2}$, Amanda Chávez V. ${ }^{3}$, Hermelinda Rivera G. ${ }^{3}$ \\ y Wilfredo Huanca L. ${ }^{4}$
}

\section{Abstract}

\begin{abstract}
The objective of this study was to determine the prevalence of toxoplasmosis (Toxoplasma gondii) in an alpaca farm in the southern part of Peru. Blood samples of 278 alpacas (68 male and 210 females) of different ages were collected and analyzed using the indirect immunofluorescense test. The prevalence was 34.5\% $\left(\mathrm{CI}_{0.95}=28.9\right.$ ? p ? 40.1\%). The prevalence was significantly affected $(\mathrm{p}<0.05)$ by sex (males: 3.0 ; females: $44.8 \%$ ) and age $(? 3$ years $=16.7 ;>3$ years $=40.8)$.
\end{abstract}

Key words : toxoplasmosis, alpaca, prevalence, immunofluorescence, serology

La toxoplasmosis producida por el Toxoplasma gondii constituye la zoonosis parasitaria más frecuente. Se estima que el60\% de personas son reactoras a esta parasitosis a nivel mundial (Amato Neto et al., 1995). La ocurrencia de toxoplasmosis ha estado mayormente ligada a cuadros de carácter subclínico, y el interés de esta parasitosis estaba circunscrita a problemas de mortalidad neonatal y abortos, siendo muy baja la frecuencia de casos clínicos (Frenkel, 1971; Scott, 1978); sin embargo, con el advenimiento del Síndrome de Inmunodeficiencia Adquirida (SIDA), esta protozoonosis se ha convertido en motivo de preocupación por parte de las autoridades sanitarias internacionales de salud. Estas instituciones recomiendan actual- mente la revisión de los aspectos epidemiológicos, patogenia, diagnóstico y tratamiento de la toxoplasmosis, por haberse constituido en una de las más importantes y frecuentes enfermedades oportunistas asociadas con el SIDA. En este contexto, las especies de animales domésticos de consumo humano han adquirido gran importancia, dado que la principal forma de transmisión de la toxoplasmosis hacia el hombre, está representada por el consumo de carne infectada insuficientemente cocida.

En el país, los camélidos sudamericanos juegan un rol muy importante en la economía de las regiones altoandinas, constituyendo su carne la fuente principal de

\footnotetext{
${ }^{1}$ Laboratorio de Medicina Veterinaria Preventiva, FMV-UNMSM

E-mail: francisco suarez2001@ hotmail.com

${ }^{2}$ Práctica privada

${ }^{3}$ Laboratorio de Microbiología y Parasitología Veterinaria, FMV-UNMSM

${ }^{4}$ Laboratorio de Reproducción Animal, FMV-UNMSM
} 
Cuadro 1. Prevalencia de Toxoplasma gondii en alpacas del Centro de Investigación IVITA-Maranganí. 2002

\begin{tabular}{rrrrrrrrr}
\hline & \multicolumn{9}{c}{ Resultado } & & \multicolumn{2}{c}{ IC $(\%)^{1}$} \\
\cline { 2 - 3 } & Positivo & Negativo & Total & $\%$ & & Inferior & Superior \\
\hline Sexo & & & & & & & & \\
& Macho & 2 & 66 & 68 & 2.9 & & 0.0 & 7.0 \\
& Hembra & 94 & 116 & 210 & 44.8 & & 38.0 & 51.5
\end{tabular}

Edad

\begin{tabular}{rcccccc} 
? 3 años & 12 & 60 & 72 & 16.7 & 8.1 & 25.3 \\
$>3$ años & 84 & 122 & 206 & 40.8 & 34.1 & 47.5 \\
\hline Total & 96 & 182 & 278 & 34.5 & 28.9 & 40.1 \\
\hline
\end{tabular}

${ }^{1}$ Intervalo de confianza del $95 \%$

proteína en la dieta del campesino. Por otro lado, la frecuencia de abortos en rebaños de camélidos constituye un problema latente y se especulaqueelToxoplasma gondii sea uno de los agentes responsables de la toxoplasmosis. Existen escasos estudios realizados en camélidos sudamericanos sobre esta enfermedad en el país; así, Leguía et al. (1998) sugieren que el 50\% de alpacas son reactores a la prueba de hemaglutinación indirecta. Estudios mas recientes, como los de Pastor (2002) en vicuñas y Gómez (2002) en alpacas indican seroprevalencias de $15 \mathrm{y}$ $45 \%$, respectivamente.

Se realizó un estudio en la unidad de producción de alpacas de la Estación Experimental del Centro de Investigación IVITAMaranganí en los meses de enero y febrero del 2002. Se tomaron muestras de sangre a 278 alpacas (210 hembras y 68 machos) de diferentes edades.

Los sueros se obtuvieron por centrifugación y se transportaron al Laboratorio de Parasitología de la Facultad de Medicina Veterinaria, UNMSM, donde se almacenaron a $-20^{\circ} \mathrm{C}$ hasta su análisis por la prueba de inmunofluorescencia indirecta, para detectar la presencia de anticuerpos contra $T$. gondii. La determinación de positividad fue de carácter cualitativo.

La prevalencia se analizó de acuerdo a su frecuencia de presentación (sueros positivos a la prueba serológica), expresándose en forma porcentual. El intervalo de confianza del $95 \%$ se calculó mediante la aproximación normal a la distribución binomial (Norman y Streiner, 1996). Se analizaron las variables sexo y edad, empléandose los intervalos de confianza para determinar la existencia de significancia estadística, así como la prueba de chi cuadrado para establecer la existencia de asociación entre las variables indicadas (Daniel, 2002).

En el Cuadro 1 se muestran los resultados de la prueba de inmunofluorescencia indirecta aplicada a los sueros de las alpacas, observándose una prevalencia general de $34.5 \%$. No obstante que la prevalencia encontrada es inferior al 50\% reportado por Leguía et al. (1988) en alpacas de una empresa ganadera de Puno, las diferencias son mínimas si observamos los intervalos de confianza. Además, se debe considerar las di- 
ferencias entre las técnicas utilizadas, ya que en aquel estudio se empleó la prueba de hemaglutinación indirecta, que ofrece menor especificidad, en comparación con la prueba de inmunofluorescencia indirecta. Larsson (1976) y D' Angelino (1983) indican que las diferencias de los valores asignados a las prevalencias de toxoplasmosis se deben a las técnicas utilizadas y a la falta de estandarización de los antígenos empleados en las pruebas serológicas.

La mayor prevalencia de reactores a toxoplasmosis en alpacas hembras en comparación a estudios similares (Rojas et al., 1993) es difícil de explicar, aunque es posible que se deba a las diferencias en el manejo de los animales. Sin embargo, se tiene evidencia de diferencias entres sexos en cerdos (Montoya $e t$ al., 1981; Bustamante y Suárez, 2000).

Los resultados de los sueros de alpacas, según edad de los animales, se muestran en el Cuadro 1. Los animales mayores de 3 años presentaron una mayor prevalencia $(40.8 \%)$ que los animales de hasta 3 años (16.7\%) $(\mathrm{p}<0.05)$

Los hallazgos del presente estudio, corroboraron los resultados reportados por Leguía et al. (1988), quienes encontraron una relación positiva entre la prevalencia de reactores a toxoplasmosis con la edad en alpacas. Este fenómeno acontece en todas las especies de animales, así Amato Neto et al. (1995) encontró en seres humanos que la proporción de individuos positivos a pruebas serológicas tienden a incrementarse progresivamente con la edad, igualmente Freire $e t$ al. (1991), hallaron en perros un aumento de reactores con la edad.

Se concluye que las alpacas de la Estación Experimental del Centro de Investigación IVITA-Maranganí presentan una prevalencia de $34.5 \%$ a Toxoplasma gondii, observándose una mayor frecuencia en hembras y en animales de mayor edad.

\section{Literatura Citada}

1. Amato Neto V.; E.A. Servolo; G.C. Levi; M.I. Seixas. 1995. Toxoplasmose. Ed. Sarvier. Sao Paulo, Brasil.

2. Bustamante, J.; F. Suárez. 2000. Estudio comparativo de frecuencias de toxoplasmosis en porcinos procedentes de crianza tecnificada y no tecnificada. Rev. Inv. Vet. Perú 11: 32-39.

3. Daniel, W.W. 2002. Bioestadística: Base para el análisis de las ciencias de la salud. $4^{a}$ ed. Ed. LIMUSA. México. 755 p.

4. Freire, R.L.; I.T. Navarro; O. Vidotto; C.C. Vianna. 1991. Prevalência de anticorpos anti-Toxoplasma gondii en cães atendidos no hospital veterinário da UEL-PR. Rev. Parasitol. 1: 18.

5. Frenkel, J.K. 1971. Toxoplasmosis: mechanisms of infection, laboratory, diagnosis and management. Curr. Top. Pathol. 54: 28-75.

6. Gómez, F. 2002. Determinación de la seroprevalencia de toxoplasmosis en alpacas y llamas en la Estación Experimental INIA-Puno. Tesis de Médico Veterinario. Facultad de Medicina Veterinaria, Univ. Nacional Mayor de San Marcos. Lima. 56 p.

7. Leguía, G.; H. Samamé; C. Guerrero; M. Rojas; A. Núñez. 1998. Prevalencia de anticuerpos contra Toxoplasma gondii en alpacas. Rev. Camel. Sud. IVITA 6: 19-22.

8. Montoya, F; L. Ramírez; A. Loaiza; J. Henao; G. Murillo. 1981. Prevalencia de anticuerpos para Toxoplasma gondii en bovinos y porcinos. Boletín de la Oficina Sanitaria Panamericana 91: 219-226.

9. Norman, G.R.; D.L. Streiner. 1996. Bioestadística. Mosby \& Doyma Libros. Madrid, España. 272 p.

10. Pastor, J. 2002. Seroprevalencia de Toxoplasma gondii en vicuñas de Puno. Tesis de Médico Veterinario. Facultad de Medicina Veterinaria, Univ. Nacional Mayor de San Marcos. Lima. 41 p. 
11. Rojas M.; I. Lobato; M. Montalvo. 1993. Prevalencia de Toxoplasma gondii en camélidos sudamericanos.
Rev. Inv. Pec. IVITA 6: 22-27.

12. Scott, R.J. 1978. Toxoplasmosis. Bull. Hyg. Trop. Dis. 75: 809-827. 